\title{
A blow-up problem for a nonlinear heat equation in the complex plane of time
}

\author{
C.-H. Cho' ${ }^{1}$ - H. Okamoto ${ }^{2} \cdot$ M. Shōji ${ }^{3}$
}

Received: 21 February 2015 / Revised: 28 October 2015 / Published online: 14 December 2015

(C) The Author(s) 2015. This article is published with open access at Springerlink.com

\begin{abstract}
Solutions of a nonlinear heat equation are numerically computed in the time variable $t$ lying in the complex plane, and possible singularities are sought. It turns out that in the complex half plane $\{\Re[t] \geq 0\}$, where $\Re$ denotes the real part of a complex number, there is no singularity other than that which exists on the real line. However, if we compute further in the Riemann surface, new singularities are found. A certain nonlinear Schrödinger equation which is associated with our problem is also computed numerically and we propose a conjecture that it is well-posed globally in time.
\end{abstract}

Keywords Nonlinear heat equation $\cdot$ Singularity $\cdot$ Ill-posed problem

Mathematics Subject Classification $\quad 35 \mathrm{~K} 55 \cdot 35 \mathrm{R} 30$

\section{Introduction}

We consider blow-up solutions of a nonlinear heat equation in the following setting of the initial-boundary value problem:

$$
\begin{aligned}
u_{t} & =u_{x x}+u^{2} & & (0<x<1), \\
u(0, x) & =u_{0}(x) & & (0<x<1),
\end{aligned}
$$

$凶$ H. Okamoto okamoto@kurims.kyoto-u.ac.jp

Department of Mathematics, National Chung Cheng University, Chia-Yi 621, Taiwan, ROC

2 Research Institute for Mathematical Sciences, Kyoto University, Kyoto 606-8502, Japan

3 Department of Mathematical and Physical Sciences, Japan Women's University, 2-8-1 Mejirodai, Bunkyo-ku, Tokyo 112-8681, Japan 
with the periodic boundary condition in $x$. Here, the subscripts $t$ and $x$ imply differentiation. As for the initial data, we assume that $u_{0}$ is continuous, $u_{0}(x) \geq 0$ everywhere and $u_{0} \not \equiv 0$. It is well-known that the solution blows up in finite time. Namely, the $L^{\infty}$-norm $\|u(t)\|_{L^{\infty}}$ tends to $\infty$ as $t$ tends to a certain $T<\infty$. Here and hereafter $u(t)$ denotes $u(t, \cdot)$. Blow-up problems for nonlinear heat equations are studied by many researchers and references are abundant. See $[9,10,25,29,35]$ for instance.

The purpose of the present paper is to study this problem for complex $t$ with $\Re[t] \geq 0$ and to show some interesting phenomena which are quite different from those with real $t$. In particular, we will show by numerical experiments that the blow-up disappears by a perturbation into upper and lower quadrants of the complex plane.

If we extend the time variable into a complex plane, then $u$, as well as $t$, becomes necessarily complex-valued. Masuda [26,27] considered (1), (2) in the complex plane with $\Re[t]>0$ with the Neumann boundary condition and proved that, if the initial data is close to a constant, a time-global solution is possible in the shaded domain of Fig. 1a, which bypasses the real singularity and extends to infinity. He showed also that the existence of an analytic solution is possible in the mirror-image of the domain in Fig. 1a about the real axis. Further, if the solution agrees in the intersection of the two domains, the initial data is a constant function. Namely, a non-constant solution can be analytically continued into the complex plane but is no longer a single-valued function in real $t>T$, where $T$ is the real blow-up time. His result implies that the singularity is not an isolated singularity but a branching point.

Masuda's pioneering work does not seem to have a follower. In fact if we wish to add anything new in a rigorous way, we encounter a serious difficulty. For instance, we tried unsuccessfully to prove global existence without assuming closeness to a constant. His paper seems to be a good example of a paper of high originality with few citations. It seems to us that, although there are numerous papers studying blow-up of solutions of nonlinear parabolic equations, few investigate singularities in the complex $t$-plane. Our purpose in this paper is to study solutions by a numerical method and to generalize the result of Masuda. One of our results is an interesting conjecture about a certain nonlinear Schrödinger equation, which will be explained in Sect. 4.

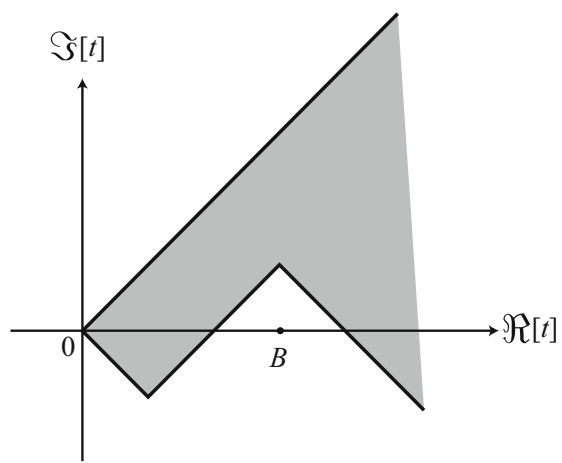

(a)

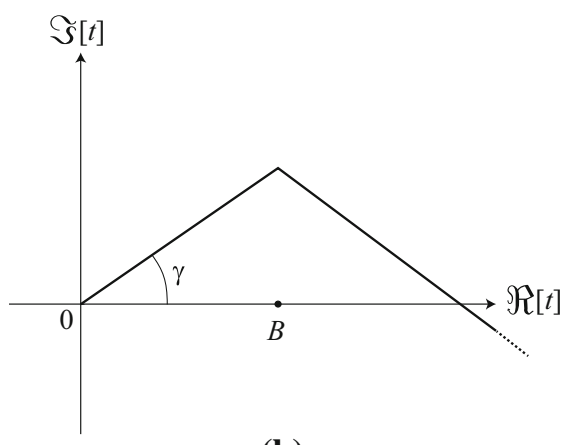

(b)

Fig. 1 a Masuda's unbounded domain of well-posedness consisting of two semi-infinite straight lines and two finite line-segments. $B$ denotes the point where $u$ blows up. b The path $\tilde{\Gamma}_{\gamma}$ 
There are similar but different studies of singularities. Sulem et al. [33] investigated numerically singularities of solutions of various evolution equations in the complex $x$ plane. They showed that complex singularities do exist in many equations. The authors of [13] considered a nonlinear heat equation where $u$ is complex-valued but both $t$ and $x$ are real-valued. These works might have a relation with ours. In particular, the theory of [13] might be applied for proving our conjectures.

Search for a singularity of solutions of nonlinear differential equations in the complex $t$-plane is not new, either. For instance, Kimura and Pelz [22] computed numerically the Navier-Stokes equations in the complex $t$-plane. Their result seems to show that there is no singularity in $\Re[t]>0$. [1] and [31] considered the Euler equations for incompressible inviscid fluid in complex $t$ and possible singularities are reported. Singularities in these equations are difficult to detect. Complex singularities of solutions of two-dimensional Euler equations are considered in Pauls et al. [28]. On the other hand, the ordinary differential equations describing the motion of point vortices do have singularities on the real $t$ axis: see [20,21]. In some cases, if a perturbation is added to the system or the initial data, the singularity disappears from the real axis and moves to the complex $t$-plane: [20,21].

We would like to know what kind of singularity is possible for (1) in the complex $t$-plane. Since the analytic function $t \mapsto u(t, \cdot)$ from the complex plane to a certain function space, e.g. $L^{2}$ or the Sobolev space $H^{1}$, is determined by its natural boundary (in the sense of a defining domain of an analytic function), singularities, if we know them all in the domain of definition, determine the function. Thus our question naturally arises.

The present paper consists of seven sections. We explain our numerical method in Sect. 2. In Sect. 3 we present the result of our numerical experiments in the case of $\Re[t]>0$. Section 4 is devoted to the case of $\Re[t]=0$. In Sect. 5 we look for singularities in the extended domain of definition of the analytic function. An inverse problem is considered in Sect. 6. Section 7 is for concluding remarks.

\section{Finite difference scheme}

Finite difference schemes have been designed for parabolic blow-up problems, see [4-8] and the references therein. We use the idea in these papers. More specifically we use the following scheme in [7]: To apply a finite difference scheme, we choose grid points $\left\{x_{k}\right\}$ defined by

$$
x_{k}=k h \quad(0 \leq k \leq K)
$$

where $K$ is a positive integer and $h=1 / K$. The parameter $\tau>0$ is chosen so that $\tau / h^{2}<1 / 2$. While $u$ is not very large, we compute values of $u$ at $x=x_{k}$ and $t_{n}=n \tau$ for $0 \leq k \leq K$ and $n=1,2, \ldots$. If $u$ becomes large, we define the time increment adaptively. The scheme can be written in the following way:

$$
\frac{u_{k}^{n+1}-u_{k}^{n}}{\Delta t_{n}}=\frac{u_{k-1}^{n}-2 u_{k}^{n}+u_{k+1}^{n}}{h^{2}}+\left(u_{k}^{n}\right)^{2}, \quad u_{j}^{n}=u_{j^{\prime}}^{n}\left(j=j^{\prime} \bmod (K)\right),
$$


where $\Delta t_{n}$ are defined with a constant $c>0$ as follows:

$$
\Delta t_{n}=\min \left\{\tau, \frac{c}{\left\|u^{n}\right\|_{\infty}}\right\} \quad\left(\left\|u^{n}\right\|_{\infty}=\max _{1 \leq k \leq K}\left|u_{k}^{n}\right|\right) .
$$

Then $t_{n}$ is defined by $t_{n}=\sum_{j=0}^{n-1} \Delta t_{j}$ and it is expected that $u_{k}^{n}$ approximates $u\left(t_{n}, x_{k}\right)$. It is proved in [7] that $\sum_{n=0}^{\infty} \Delta t_{n}<\infty$, and this value is, by definition, the approximate blow-up time.

We first compute a solution of real $t$ by this scheme to obtain the approximate blow-up time. Accordingly we can obtain the approximate position of the point $B$ in Fig. 1 . We then compute the solution along the following path $\Gamma_{\gamma}$ and $\tilde{\Gamma}_{\gamma} . \Gamma_{\gamma}$ is defined as the following straight line: $\rho e^{\mathrm{i} \gamma} \quad(0 \leq \rho<\infty)$, where $\gamma$ is a real number $\in(-\pi / 2, \pi / 2)$. The path $\tilde{\Gamma}_{\gamma}$ is defined as

$\rho e^{\mathrm{i} \gamma}\left(0 \leq \rho \leq \frac{T}{\cos \gamma}\right), \quad e^{\mathrm{i} \gamma} \frac{T}{\cos \gamma}+e^{-\mathrm{i} \gamma}\left(\rho-\frac{T}{\cos \gamma}\right) \quad\left(\frac{T}{\cos \gamma} \leq \rho<\infty\right)$,

where $\mathrm{i}=\sqrt{-1}, T$ is the approximate blow-up time, and $\gamma$ is as above: see Fig. 1b. There is no particular meaning of the choice of $\tilde{\Gamma}_{\gamma}$. Other choices would be equally possible. We took it just for convenience.

Let us first take the straight path $\Gamma_{\gamma}$ and consider the equation along this path. Namely,

$$
\frac{1}{e^{\mathrm{i} \gamma}} u_{\rho}=u_{x x}+u^{2} \quad(0<x<1,0<\rho) .
$$

We consider this equation with the same initial data and the same boundary condition.

Let us take a uniform time increment $\tau$. We then consider the following scheme:

$$
\frac{u_{k}^{n+1}-u_{k}^{n}}{e^{\mathrm{i} \gamma} \tau}=\frac{u_{k-1}^{n}-2 u_{k}^{n}+u_{k+1}^{n}}{h^{2}}+\left(u_{k}^{n}\right)^{2}, \quad u_{j}^{n}=u_{j^{\prime}}^{n} \quad\left(j=j^{\prime} \bmod (K)\right),
$$

which may be written as

$$
u_{k}^{n+1}=\left(1-2 \lambda e^{\mathrm{i} \gamma}\right) u_{k}^{n}+\lambda e^{\mathrm{i} \gamma}\left(u_{k-1}^{n}+u_{k+1}^{n}\right)+\tau e^{\mathrm{i} \gamma}\left(u_{k}^{n}\right)^{2},
$$

where $\lambda=\tau / h^{2}$.

We now analyze the stability by von Neumann's method, i.e., we forget about the boundary and we assume as if $k$ extends from $-\infty$ to $+\infty$. We also discard the nonlinear term. Then we have

$$
\frac{u_{k}^{n+1}-u_{k}^{n}}{e^{\mathrm{i} \gamma} \tau}=\frac{u_{k+1}^{n}-2 u_{k}^{n}+u_{k-1}^{n}}{h^{2}},
$$

which is written as

$$
u_{k}^{n+1}=\left(1-2 \lambda e^{\mathrm{i} \gamma}\right) u_{k}^{n}+\lambda e^{\mathrm{i} \gamma}\left(u_{k-1}^{n}+u_{k+1}^{n}\right) .
$$


Substitute now $u_{k}^{n}=\exp (\mathrm{i} \beta k)$, where $\beta$ is any real number, into the right hand side of (8). We then obtain

$$
\left[\left(1-2 \lambda e^{\mathrm{i} \gamma}\right)+\lambda e^{\mathrm{i} \gamma} \times 2 \cos \beta\right] e^{\mathrm{i} \beta k} .
$$

Von Neumann's condition for the stability is obtained if we require that the absolute value of the square bracket is $\leq 1$ for all $\beta \in \mathbb{R}$. After some calculation, the condition turns out to be $\lambda(1-\cos \beta) \leq \cos \gamma$. This is required to hold true for all $\beta \in \mathbb{R}$. Therefore we have

Theorem 1 If $\lambda=\tau / h^{2} \leq \frac{1}{2} \cos \gamma$, then the scheme (7) is stable in the sense of von Neumann.

Although we do not know if this is already known in this form, it is very possible that its contents may be known for experts (see, for instance, [3,24,30]).

We now carry out more technical stability analysis and consider (7) with the periodic boundary condition $u_{j}^{n}=u_{j^{\prime}}^{n} \quad\left(j=j^{\prime} \bmod (K)\right)$.

We define the following discrete norms:

$$
\left\|v^{n}\right\|_{2}^{2}=h \sum_{k=0}^{K-1}\left|v_{k}^{n}\right|^{2}, \quad\left\|v_{x}^{n}\right\|_{2}^{2}=h \sum_{k=0}^{K-1}\left|\frac{v_{k+1}-v_{k}}{h}\right|^{2} .
$$

We can prove the following theorem, which asserts the $H^{1}$-stability under the assumption which is just a little stronger than that in Theorem 1 .

Theorem 2 Assume that $-\pi / 2<\gamma<\pi / 2$. Suppose that $\lambda=\tau / h^{2} \leq \frac{2-\tau}{4} \cos \gamma$. Then the solution of (7) satisfies

$$
\left\|u^{n+1}\right\|_{2}^{2} \cos \gamma+\left\|u_{x}^{n+1}\right\|_{2} \leq\left\|u^{n}\right\|_{2}^{2} \cos \gamma+\left\|u_{x}^{n}\right\|_{2} \quad(n=0,1,2, \ldots),
$$

where norms are defined by (9).

Proof requires a careful manipulation of elementary inequalities. Since it is rather tedious, we omit it. Note that Theorem 1 gives us a necessary condition, while Theorem 2 does a sufficient condition.

We now consider (5).

Theorem 3 Assume that $-\pi / 2<\gamma<\pi / 2$ and $\lambda<\frac{2-\tau}{4} \cos \gamma$. Given $T_{0}>0$, suppose that the solution of (1) and (2) is smooth in $0 \leq t \leq T_{0}$. Then, for sufficiently small $h$,

$$
\left\|u_{k}^{n}-u\left(n \tau, x_{k}\right)\right\|_{2} \leq c h^{2}
$$

as long as $n \tau \leq T_{0}$. Here, $\left\{u_{k}^{n}\right\}$ is the solution of (5), the norm is defined by (9), and $c$ is a constant which depends on $u_{0}$ and $T_{0}$ but is independent of $h$ and $\tau$.

Again, the proof is omitted, since it is standard (though is not short): Techniques for proving these theorems are standard, see for instance [32]. 


\section{Experiments}

We now present our experimental results.

We first consider the case where $t$ is real. We choose $u_{0}(x)=50(1-\cos 2 \pi x)$ $(0 \leq x \leq 1)$ as an initial data, and set $\tau=0.00001$ and $K=200$, whence $\lambda=0.4$, to use (3). We obtained Fig. 2 . The solution blows up at approximately $T \approx 0.0119$.

With this information in hand, we next compute the solution in the complex plane. Since the symmetry $u\left(\rho e^{-\mathrm{i} \gamma}, x\right)=\overline{u\left(\rho e^{\mathrm{i} \gamma}, x\right)}$ for a real $\rho$ is obvious, we compute it only in $\mathfrak{R}[t] \geq 0, \mathfrak{\Im}[t] \geq 0$, where $\Re$ and $\Im$ denote the real and imaginary parts of a complex number, respectively. We first compute the solution on the half-lines: $\rho e^{\mathrm{i} \gamma}$ with $\gamma=15^{\circ}, 30^{\circ}, 45^{\circ}$ and $60^{\circ}$ to obtain Fig. 3, which shows how $\left\|u\left(\rho e^{\mathrm{i} \gamma}\right)\right\|_{\infty}:=$ $\max _{0 \leq x \leq 1}\left|u\left(\rho e^{\mathrm{i} \gamma}, x\right)\right|$ changes with $\rho$. In each case, the solution exists for all $\rho$ and decays to zero, although a considerable increase is admitted if $\gamma$ is small and $\rho$ is near 0.01 . The profiles of $\Re[u]$ and $\Im[u]$ are plotted in Fig. 4 in the case of $\gamma=45^{\circ}$. The imaginary part is initially zero, but it increases quickly, and the order of the magnitude

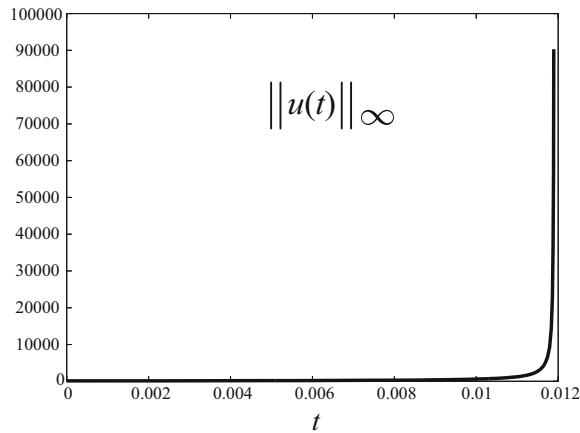

(a)

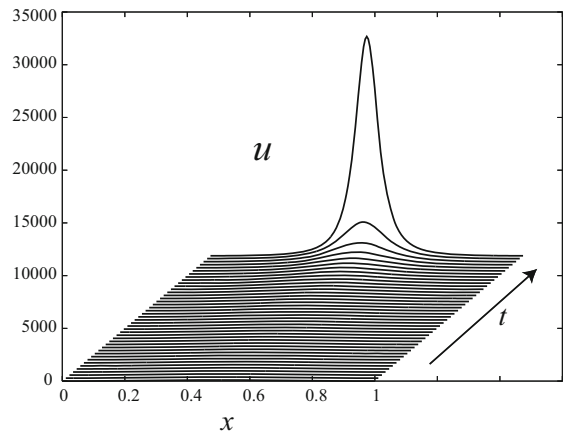

(b)

Fig. 2 a Plots of $\|u(t)\|_{\infty}:=\max _{0 \leq x \leq 1}|u(t, x)|$ versus $t$. b Graph of $u(t, x)$. Initial data is $u_{0}(x)=$ $50(1-\cos 2 \pi x) . K=200, \tau=0.00001$

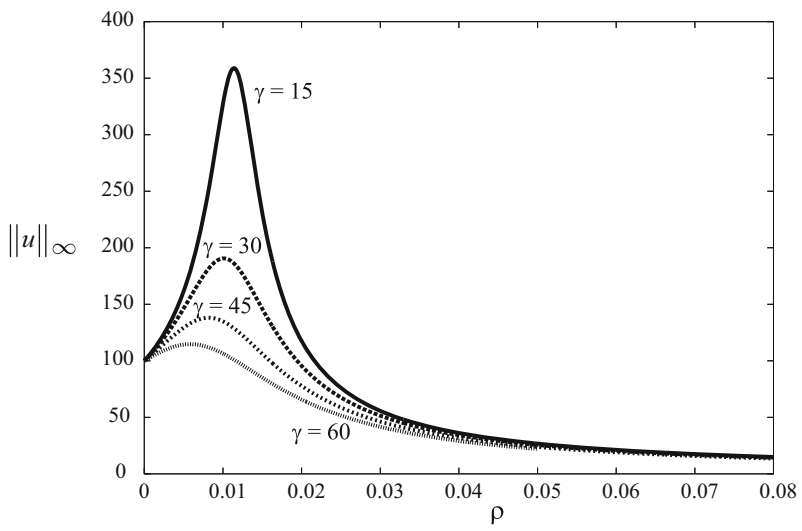

Fig. $3 \gamma=15^{\circ}, 30^{\circ}, 45^{\circ}, 60^{\circ} . \tau=0.8 \times 10^{-5}$ for $\gamma=15^{\circ}, 30^{\circ}, 45^{\circ}$ and $\tau=0.5 \times 10^{-5}$ for $\gamma=60^{\circ}$ 


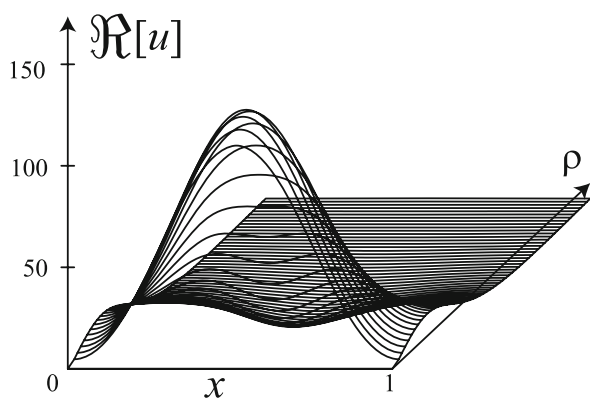

(a)

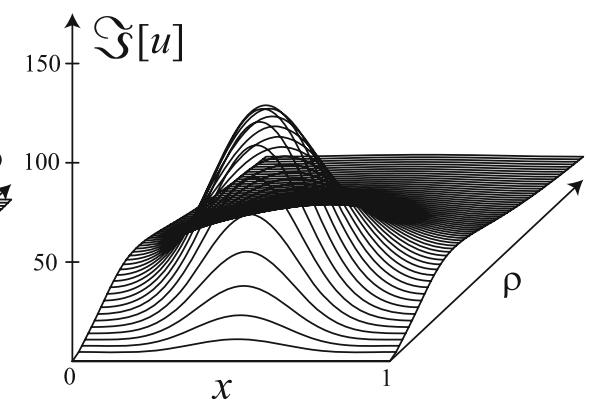

(b)

Fig. 4 Plots of $\mathbf{a} \Re[u]$ and $\mathbf{b} \Im[u]$ on $\Gamma_{\gamma}$ with $\gamma=45^{\circ}$. For $\rho$ large enough, $\Re[u]$ is negative and $\Im[u]$ is positive

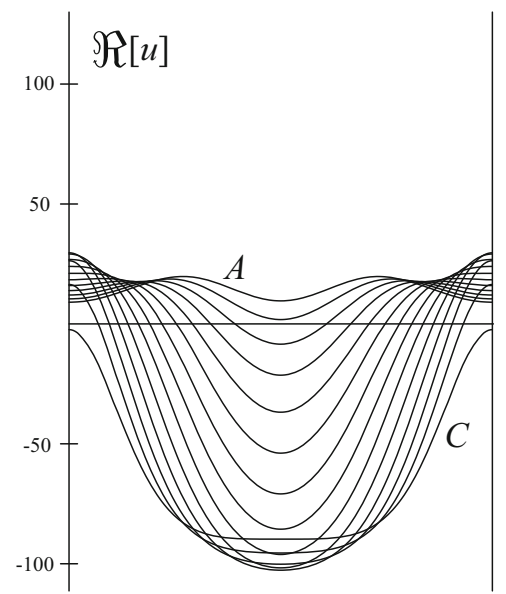

(a)

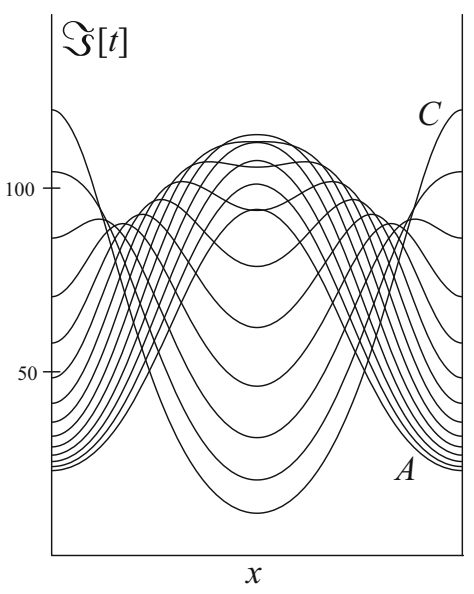

(b)

Fig. 5 Solutions on $A C$ of the path $\tilde{\Gamma}_{\pi / 4}$. a $\Re[u] . \mathbf{b} \Im[u]$

becomes comparable to the real part. Then, both parts begin to decrease. Note also that the real part is negative everywhere for large $\rho$ and the imaginary part becomes positive. Qualitatively the same pictures are obtained for other $\gamma$ (figure omitted).

Figure 5 shows how the real and imaginary parts of $u$ changes on the line segment $A C$ in Fig. 6. At the point $C, t$ is approximately 0.0238 , which is twice the blow-up time. From there we may continue the computation along the path above, $(C E$ of Fig. 6) or we may turn the path and compute along the real axis ( $C D$ of Fig. 6), or we can proceed vertically downward ( $C F$ of Fig. 6). (But in the last case the explicit finite difference scheme becomes unstable and we have to use a different one. See the next section.) In all the three cases, our computations show that the solution does not blow up and tends to zero as $|t| \rightarrow \infty$, although in the course of time the solution can be bigger and has as many peaks as five. The solution in this region will be discussed later in Sect. 5. 
Fig. 6 Paths of integration

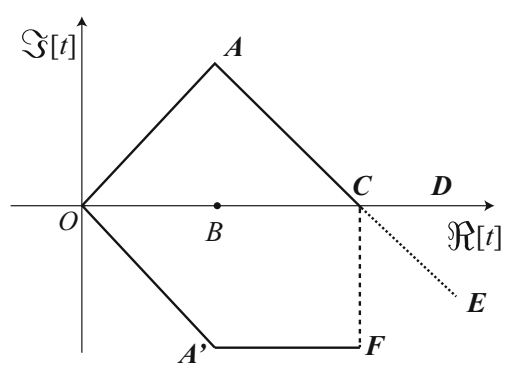

We computed on other paths with different inclination to find similar behavior of the solution. We do not feel it necessary to draw figures for these results since they are broadly similar.

\section{Pure imaginary time}

If $t$ is replaced by is with $\mathrm{i}=\sqrt{-1}$ and $s \in \mathbb{R}$, (1) becomes

$$
\frac{1}{\mathrm{i}} u_{s}=u_{x x}+u^{2} \quad(0<x<1) .
$$

This is a nonlinear Schrödinger equation.

The well-posedness of the nonlinear Schrödinger equation is studied well. For our purpose it is enough to consider it in the Sobolev spaces of $L^{2}$-type. The wellposedness in $L^{2}$ begins with Tsutsumi's pioneering work [34], which was generalized in many ways. For instance, Kato's theorem in [19] is easy to use: It says that for spatial dimension $m=1,2,3$ and for all $u_{0} \in L^{2}\left(\mathbb{R}^{m}\right)$, there is an $S>0$ depending only on $\left\|u_{0}\right\|_{L^{2}}$ such that a unique solution exists in $0 \leq s \leq S$ with $u(0)=u_{0}$ for

$$
u_{s}=\mathrm{i}(\triangle u+f(u))
$$

with a certain class of $f$ including $f(u)=u^{2}$. Those results are proved when the spatial domain is the whole Euclidean space. The authors could not find a reference which explicitly states local existence of (11) in a bounded domain.

Note further that the global existence results for the nonlinear Schrödinger equation is established mostly in the case where the nonlinear term is $|u|^{p-1} u$ with $p>1$ (see [34]) or that the initial data is small (see, e.g., [2,14-16]). The blow-up results in the case of a bounded domain are available in [36]. These results, however, do not apply directly to (10).

Since we consider only a smooth initial data satisfying the boundary condition, it is enough for our purpose to use the following rather weak theorem:

Theorem 4 Let $u_{0} \in H^{2}\left(\mathbb{T}_{1}\right)$. Then, under the periodic boundary condition, there exists a unique solution of (10) in $0 \leq s \leq S$, where $S>0$ depends only on $\left\|u_{0}\right\|_{H^{2}}$. 
Here $\mathbb{T}_{1}$ denotes the one-dimensional torus (or the circle) obtained by identifying 0 and 1 of the interval $[0,1]$. Theorem 4 can be proved in a standard way. For instance the theorem by Kato in [18] can be used.

Since the finite difference scheme in the previous section is no longer stable in the present case, we consider the following implicit scheme:

$$
\frac{u_{k}^{n+1}-u_{k}^{n}}{\mathrm{i} \tau}=\frac{u_{k-1}^{n+1}-2 u_{k}^{n+1}+u_{k+1}^{n+1}}{h^{2}}+u_{k}^{n} u_{k}^{n+1},
$$

or, equivalently,

$$
-\mathrm{i} \lambda u_{k-1}^{n+1}+\left(1+2 \mathrm{i} \lambda-\mathrm{i} \tau u_{k}^{n}\right) u_{k}^{n+1}-\mathrm{i} \lambda u_{k+1}^{n+1}=u_{k}^{n} .
$$

If the periodic boundary condition is taken into account, our finite difference scheme can be written as

$$
\left(\begin{array}{cccccc}
a_{0} & -\mathrm{i} \lambda & 0 & 0 & \ldots & -\mathrm{i} \lambda \\
-\mathrm{i} \lambda & a_{1} & -\mathrm{i} \lambda & 0 & \ldots & 0 \\
0 & -\mathrm{i} \lambda & a_{2} & -\mathrm{i} \lambda & \cdots & 0 \\
\cdots & \cdots & \cdots & \cdots & \cdots & 0 \\
0 & \cdots & 0 & -\mathrm{i} \lambda & a_{K-2} & -\mathrm{i} \lambda \\
-\mathrm{i} \lambda & \cdots & 0 & 0 & -\mathrm{i} \lambda & a_{K-1}
\end{array}\right)\left(\begin{array}{c}
u_{0}^{n+1} \\
u_{1}^{n+1} \\
u_{2}^{n+1} \\
\vdots \\
u_{K-2}^{n+1} \\
u_{K-1}^{n+1}
\end{array}\right)=\left(\begin{array}{c}
u_{0}^{n} \\
u_{1}^{n} \\
u_{2}^{n} \\
\vdots \\
u_{K-2}^{n} \\
u_{K-1}^{n}
\end{array}\right)
$$

where we have set

$$
a_{k}=1+2 \mathrm{i} \lambda-\mathrm{i} \tau u_{k}^{n}
$$

It is not difficult to see that the linear part is stable in the sense of von Neumann for all $\lambda>0$. Therefore, if $\tau$ is small enough and $\max _{k}\left|u_{k}^{n}\right|$ is not too large, we can expect a stable computation by this scheme.

With the same initial data as before, we obtained Fig. 7. We also computed solutions of various other initial data to obtain quite similar results. We may therefore conjecture that if $u_{0}$ is real-valued, the solution of (10) exists globally in time and decays to zero as $s$ tends to infinity. Since there seems to be no dissipation or dispersion, it is curious that the solutions decay to zero. The following consideration may be of some help: Suppose that the initial data is a constant. Then the solution of (10) remains to be a constant in $x$ for all $s>0$. Accordingly, the solution of (10) is given by

$$
u(t, x)=\frac{u_{0}}{1-\mathrm{i} u_{0} s} \quad(0 \leq s<\infty) .
$$

Unless $\Re\left[u_{0}\right]=0, \Im\left[u_{0}\right]<0$, this solution is continuous and decays to zero with the order of $O\left(s^{-1}\right)$. The above solution with $u_{0}(x)=50(1-\cos 2 \pi x)$ is computed in $0 \leq s \leq 1$ and we obtained Fig. 8. Although the solution is not a constant, we may interpret Fig. $8 \mathrm{~b}$ as indicating $\lim _{s \rightarrow \infty} s\|u(s, \cdot)\|_{\infty}=1$. 


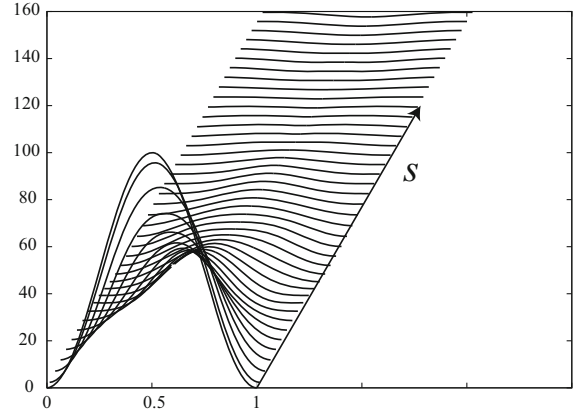

(a)

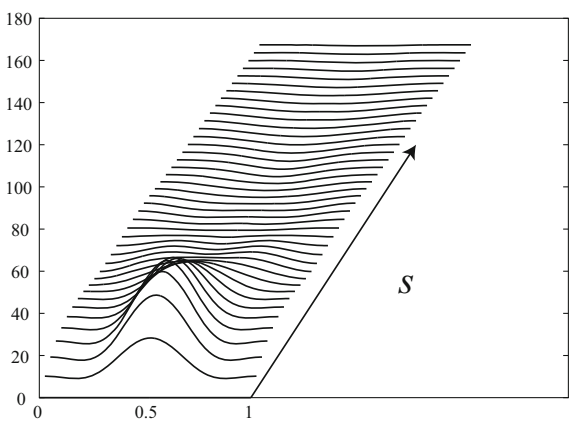

(b)

Fig. 7 The solution of (12). The real part (a) and the imaginary part (b). $u_{0}(x)=50(1-\cos 2 \pi x)$. $0 \leq s \leq 0.15, K=200$

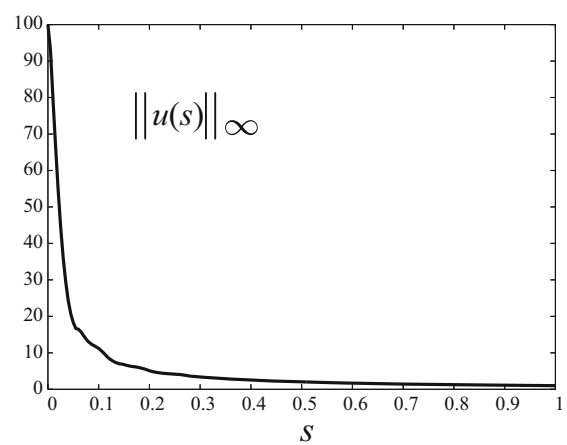

(a)

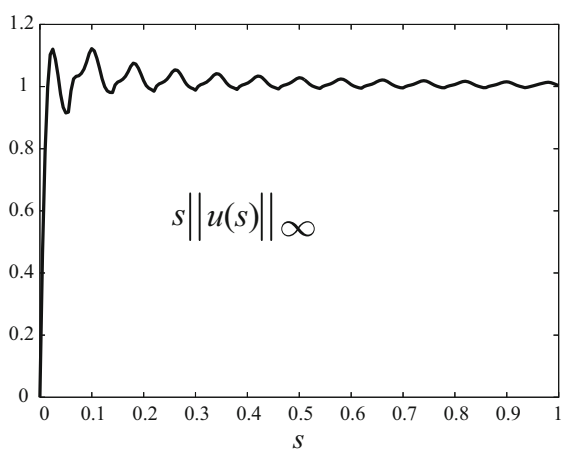

(b)

Fig. 8 The solution of (12). $u_{0}(x)=50(1-\cos 2 \pi x)$. a $\max _{0 \leq x \leq 1}|u(s, x)|$. b $s \max _{0 \leq x \leq 1}|u(s, x)|$

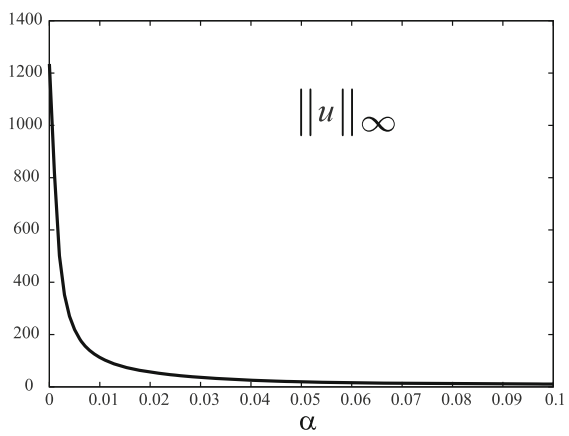

(a)

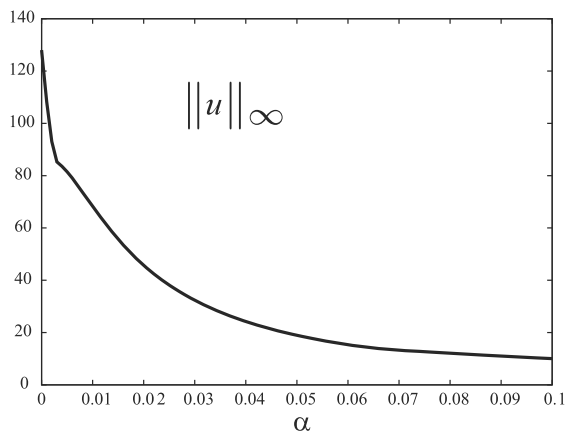

(b)

Fig. 9 a Plots of $\|u(0.011+i \alpha)\|_{\infty}$ for $0<\alpha<0.1$. b Plots of $\|u(0.0238+i \alpha)\|_{\infty}$ for $0<\alpha<0.1$

We also computed the solution of (12) with $u\left(t_{0}\right)$ as the initial data, where $t_{0}$ is any number satisfying $0<t_{0}<0.0119$. When $t_{0}=0.011$, which is rather close to the blow-up time, we obtained a solution which quickly decays to zero, see Fig. 9. 


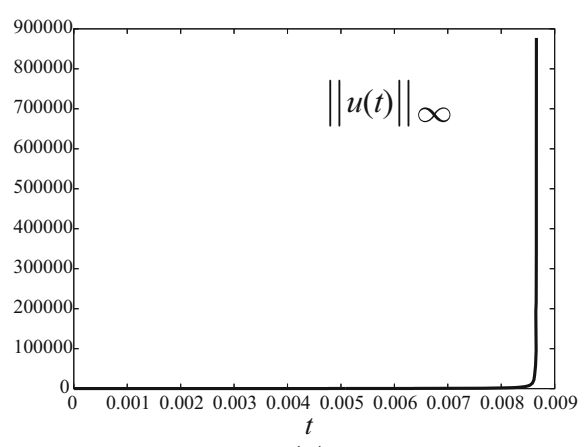

(a)

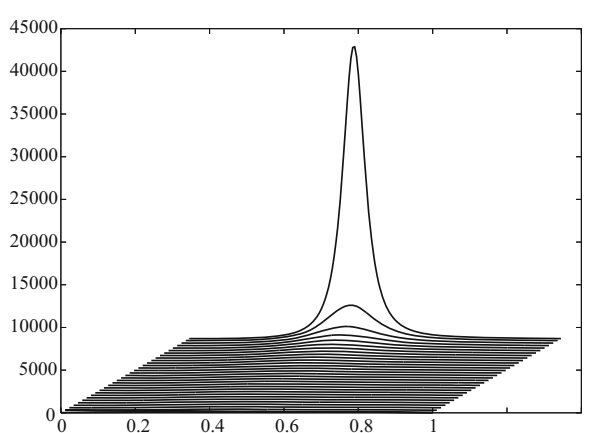

(b)

Fig. 10 Solution of (12) with (13) as the initial data

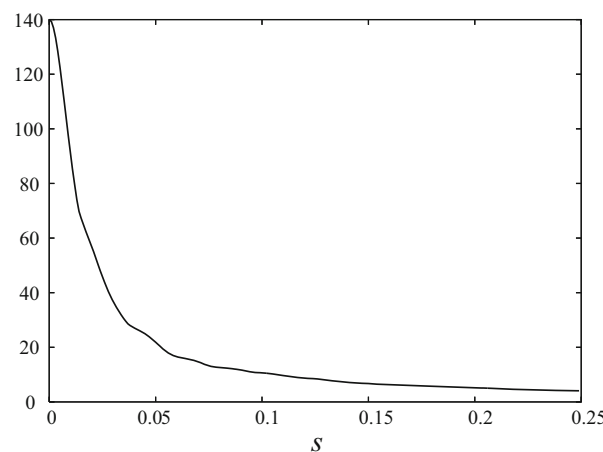

(a)

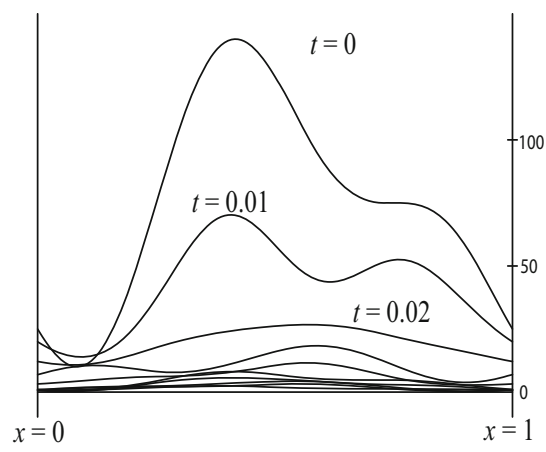

(b)

Fig. 11 (12)(13) on the imaginary axis. a $\| u($ is $) \|_{\infty}$ for $0 \leq s \leq 0.25$. b Profiles of $\Re[u]$

Similarly we obtain solutions starting from $t_{0} \in(0.0119,0.0238)$. The solution seems to decay to zero in all the cases.

We tested other initial data, too. For instance, initial data $a(1-\cos 2 \pi x)$ with various $a>0$ were tested. Also tested are asymmetric data such as

$$
u_{0}(x)=50(1-\cos 2 \pi x)+25(1-\sin 4 \pi x)
$$

which is not symmetric in $x$ about $x=1 / 2$. Let us now see if this asymmetry produces anything new. By the method as above, we found that the blow-up time is approximately 0.008658 and the blow-up point is approximately $x=0.445$. See Fig. 10. With this initial data, we solved (12) to obtain Fig. 11. Namely, the solution exists in $0 \leq s<\infty$ and decays to zero. Our result suggests that the solution decays in the order $s^{-1}$. We also tested, as initial data, $50 \cos 2 \pi x$, which has zeros in $[0,1]$, and $-50(2+\cos 2 \pi x)$, which is negative everywhere. In both cases the solution decays to zero (figure omitted).

The result of the present section seems to suggest that the solution of (10) exists for all $s$. Combined with the result in the preceding section, we may conjecture that 
our equation is well-posed in $\{t ; \mathfrak{R}[t] \geq 0$, $\Im[t] \geq 0\} \backslash\{B\}$. However, the authors are unable to prove this proposition rigorously.

\section{Singularities on the Riemann surface}

We have seen that there is no singularity in $\{t ; \mathfrak{R}[t] \geq 0, \Im[t] \geq 0\} \backslash\{B\}$, since solutions on $\Gamma_{\gamma}$ lying entirely in the upper plane are smooth for all $\gamma$ which we tested. We therefore consider a Riemann surface containing the quadrant $\{t ; \mathfrak{R}[t]>$ 0 , $\Im[t]>0\}$. See Fig. 12. In other words, we look for an analytic continuation of an analytic function $t \mapsto u(t, \cdot) \in H^{2}\left(\mathbb{T}_{1}\right)$. Once we have computed those data on the real line $0.0119<t$, we can compute solutions below this line on the Riemann surface. For instance, we compute solutions along the path $O A C F$ in Fig. 6. Note that the solution at $F$ which is computed in this way is different from the one which we compute along, say, $O A^{\prime} F$ in the lower half plane.

As a general remark, note the following facts: Let two points on the Riemann surface, say $P$ and $Q$, be given. Suppose two paths joining $P$ and $Q$ are given on the Riemann surface. Beginning from $P$, we compute $u(t, \cdot)$ along the paths to obtain the value at $Q$. If there is no singularity on the paths or in the region enclosed by the two paths, the solutions thus computed are one and the same. The converse is not necessarily true: Even if the solutions which are computed along two different paths are the same, one cannot guarantee that there is no singularity between two paths. This is because there may be an isolated singularity. Based on the following plausible argument, however, we consider an isolated singularity to be unlikely.

Consider a path starting from a regular point and ending at the singularity. Let the path be represented by $s \mapsto \eta(s) \in \mathbb{C}$ and assume that $\Re\left[\eta^{\prime}(s)\right]>0$. We then define $v(s, x)=u(\eta(s), x)$, which satisfies

$$
\frac{\partial v}{\partial s}=\eta^{\prime}(s)\left[v_{x x}+v^{2}\right]
$$

We multiply this by $\overline{v(s, x)-v_{x x}(s, x)}$, integrate the resultant by parts, and take the real part. We then obtain

Fig. 12 Schematic image of the Riemann surface
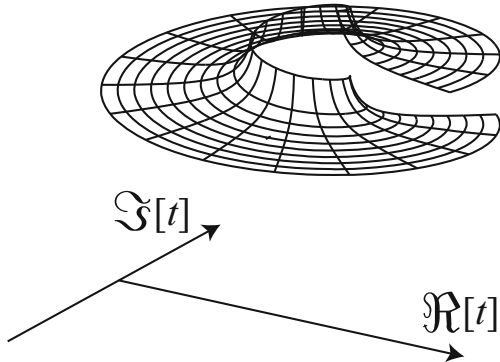


$$
\begin{aligned}
\frac{\mathrm{d}}{\mathrm{d} s} \int_{0}^{1}\left(|v|^{2}+\left|v_{x}\right|^{2}\right) \mathrm{d} x= & -2 \Re\left[\eta^{\prime}(s)\right] \int_{0}^{1}\left(\left|v_{x}\right|^{2}+\left|v_{x x}\right|^{2}\right) \mathrm{d} x \\
& +2 \Re\left[\int_{0}^{1} \eta^{\prime}(s) v\left(|v|^{2}+2\left|v_{x}\right|^{2}\right) \mathrm{d} x\right] .
\end{aligned}
$$

Since $\|v\|_{L^{\infty}}$ is bounded from above by a constant times $\sqrt{\|v\|_{L^{2}}^{2}+\left\|v_{x}\right\|_{L^{2}}^{2}}$, we get to

$$
\frac{\mathrm{d}}{\mathrm{d} s} \int_{0}^{1}\left(|v|^{2}+\left|v_{x}\right|^{2}\right) \mathrm{d} x \leq c\left(\int_{0}^{1}\left(|v|^{2}+\left|v_{x}\right|^{2}\right) \mathrm{d} x\right)^{3 / 2} \quad(0<s)
$$

where $c$ is a positive constant which depends on $\eta$ but is independent of $u$. Then with positive constant $c_{k}(k=1,2)$ we have

$$
\|v(s)\|_{H^{1}} \leq \frac{c_{1}}{c_{2}-s} .
$$

Because of the arbitrariness of the choice of $\eta$, (14) holds true on the half plane to the left of the supposed singularity.

If there is ever an isolated singularity $t_{0}$, then we may set

$$
u(t)=\sum_{n=-1}^{\infty} A_{n}(x)\left(t-t_{0}\right)^{n}
$$

where $A_{n}$ is an element of $H^{2}\left(\mathbb{T}_{1}\right)$ for all $n$. No terms of $\left(t_{0}-t\right)^{-n}(n \geq 2)$ would appear because of (14). Then, by substituting this into the differential equation, we obtain

$$
\begin{aligned}
-A_{-1} & =\left(A_{-1}\right)^{2}, \\
0 & =A_{-1, x x}+2 A_{0} A_{-1}, \\
A_{1} & =A_{0, x x}+A_{0}^{2}+2 A_{1} A_{-1}, \\
2 A_{2} & =A_{1, x x}+2 A_{-1} A_{2}+2 A_{0} A_{1}, \quad \text { etc. }
\end{aligned}
$$

We successively derive $A_{-1}=-1, A_{0}=0, A_{1}=0, A_{2}=0, \ldots$. Therefore only a constant times $\left(t-t_{0}\right)^{-1}$ is permitted among the isolated singularities even in the extended Riemann surface. Since we are dealing with a non-constant solution, only a branching singularity would be permitted.

We are well aware that this argument is not a rigorous proof, but as a matter of fact, what we obtain below are only branching points.

Let $B$ and $C$ be as above. Namely $B=T+0 \mathrm{i}, C=2 T+0 \mathrm{i}$ with $T \approx 0.0119$. Take $A^{\prime}=T-T \mathrm{i}, D=2 T-T \mathrm{i}$. The square $B A^{\prime} D C$ is now dissected into $8 \times 8=64$ small squares, see Fig. 13a. The squares are labeled as $S_{i, j}$ with $i, j=0,1, \ldots, 7$, where $i$ denotes the vertical direction (downward) and $j$ denotes the horizontal direction (to the right). Let the four corners of $S_{i, j}$ be denoted by $P_{i, j}, Q_{i, j}, M_{i, j}, N_{i, j}$, respectively 


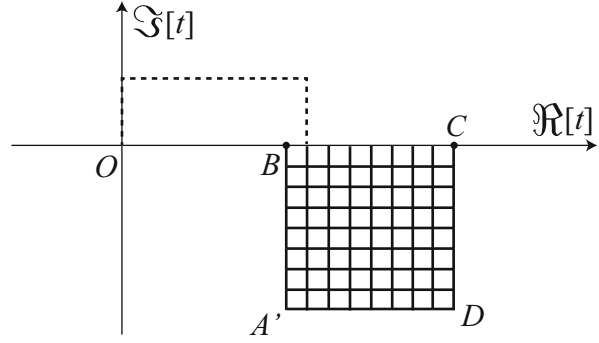

(a)

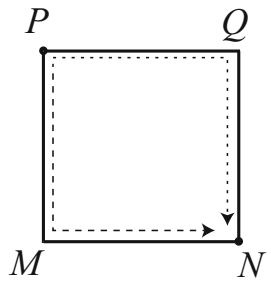

(b)

Fig. 13 Dissection of $B A^{\prime} D C$

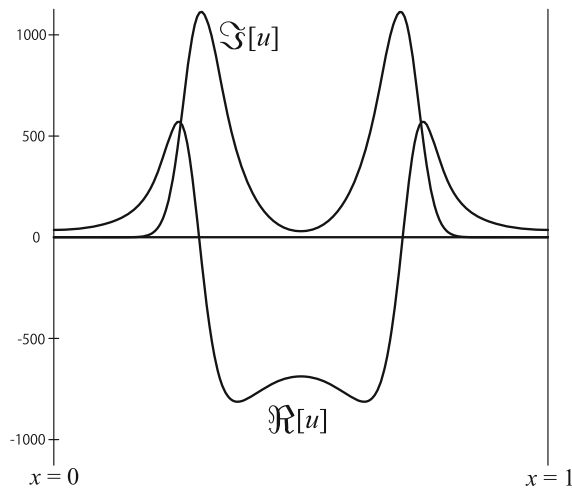

(a)

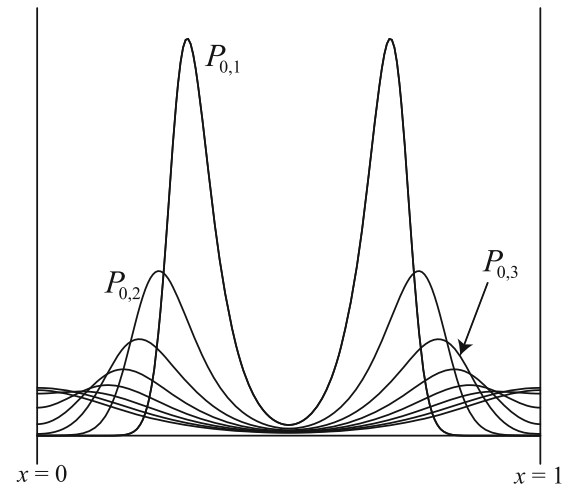

(b)

Fig. 14 a The real and imaginary parts of the solution at $P_{0,1}$. b The imaginary parts at $P_{0,1} \sim P_{0,8}$

for the left upper, right upper, left lower, and right lower corners. See Fig. 13b. By the definition, it holds that $P_{i, j}=M_{i-1, j}, Q_{i, j}=P_{i, j+1}, N_{i, j}=P_{i+1, j+1}$, etc.

We compute solutions at $P_{0, j}(j=1,2, \ldots, 8)$ by the method above. $P_{0,1}$ is computed along a path shown in Fig. 13 a by a broken line. Then $P_{0,2}$ and so forth are computed horizontally to the right. For instance, the graph at $P_{0,1}$ is drawn in Fig. 14a. From $P_{0, j}$ we compute solutions vertically downward to obtain the solution at $P_{1, j}=$ $M_{0, j}$. And then we compute horizontally to the right to $P_{1, j+1}=N_{0, j}$. Similarly we obtain a solution along the path $P_{0, j} \rightarrow Q_{0, j} \rightarrow N_{0, j}$. Thus we obtain two solutions: one which is obtained along $P_{0, j} Q_{0, j} N_{0, j}$ and the other along $P_{0, j} M_{0, j} N_{0, j}$. See Fig. 13b. If the two solutions agree at $N_{0, j}$, we draw the square $S_{0, j}$ in green, otherwise in red. We then do the same thing for $S_{1, j}$, and then $S_{2, j}$ and so on. If a red square appears, we do not compute in the squares below the red one and leave them in white. Also, if the computation diverges, we leave the square in white. These computations were carried out with $K=500$ : Since some solutions are of oscillatory nature, we needed more grid points than in the case of the previous sections.

This rule leaves ambiguity if a red square appears. Let the right lower corner of the red square be $N_{i j}$. Then the value on $P_{i+1, j+1}$ can be computed in two ways: from the above and from the left. In this case, we understand that the value computed from 
Fig. 15 Regions that two solutions agree (green) and disagree (red) (color figure online)

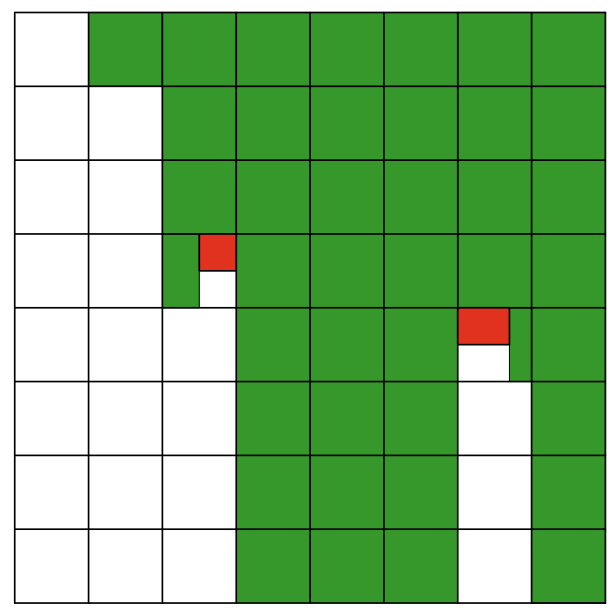

above, i.e., along $P \rightarrow Q \rightarrow N$, is taken, and solutions in $S_{i+1, j+1}$ will be computed with this rule.

After this process we obtained two red squares. We then dissected the red square into smaller squares or rectangles to test whether they belong to red or green. The result is Fig. 15. This figure strongly suggests that there are at least two branching singularities: one is in $10.5 b \leq \Re[t] \leq 11 b,-3.5 b \leq \Im[t] \leq-3 b$; the other is in $14 b \leq \Re[t] \leq 14.7 b,-4.5 b \leq \Im[t] \leq-4 b$, where $b=T / 8$. (This figure also suggests that there is another singularity in $S_{1,1}$. But we do not pursue it because of numerical difficulty which we experienced in that square.) If the reader sees Figs. 16 and 17 , the difference of the two solutions at the right lower corner of the red square would be evident. For the reader's reference we plot, in Fig. 18, the imaginary part of $u$ on a vertical line.

In addition to this square $B C D A^{\prime}$, we computed solutions also in the square $2 T \leq$ $\Re[t] \leq 3 T,-T \leq \Im[t] \leq 0$ to find no singularity there.

We also considered the case where the initial data is $u_{0}(x)=50 /(2-\cos 2 \pi x)$. This initial data is analytic but it has a singularity in the complex $x$ domain, while $u_{0}(x)=50(1-\cos 2 \pi x)$ is an entire function of $x$. Accordingly the spatial singularity may have some effects on the $t$-plane. We computed in $\mathfrak{R}[t] \geq 0, \Im[t]>0$ and no singularity was discovered. In the Riemann surface, we obtained Fig. 19: Singularities do exist but in places different from the case above. Solutions at the two lower corners are drawn in Fig. 20.

\section{Around the branching point}

The next question seems to be: Can we compute the solution in a full neighborhood of a branching point? Can we make a full turn around a branching point? For instance, can we compute the solution along the path $O \rightarrow A \rightarrow C \rightarrow A^{\prime} \rightarrow O$ ? Then the problem becomes ill-posed in Hadamard's sense, and ordinary computational methods do not work. Therefore we must use some sort of regularization and/or other means. 


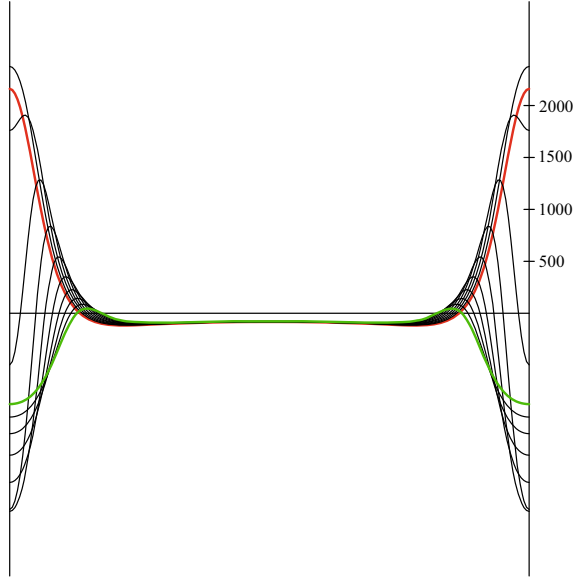

(a)

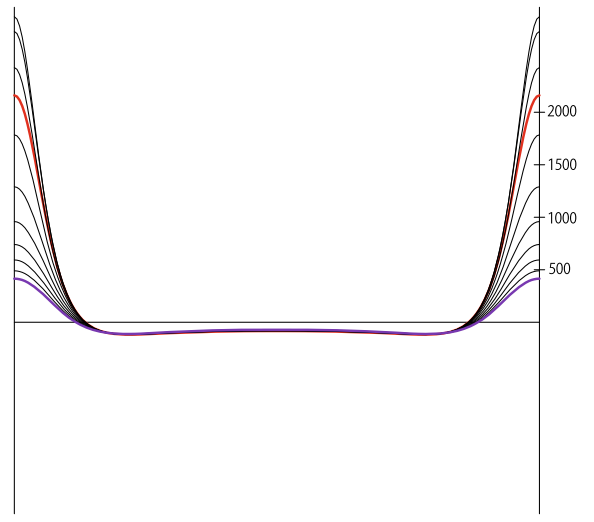

(c)

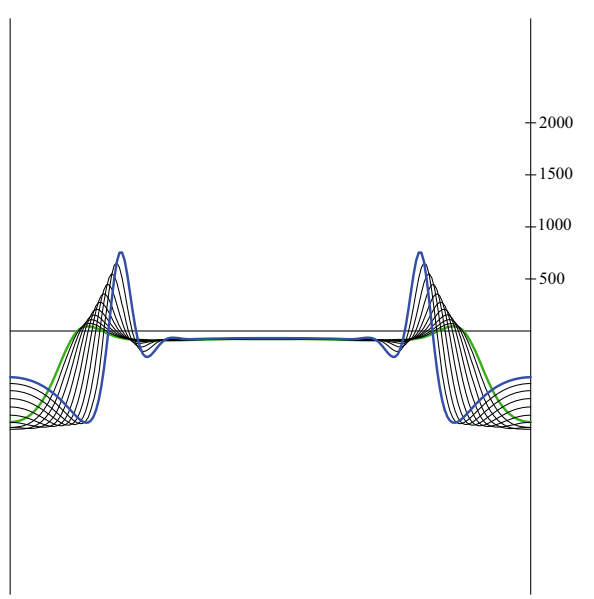

(b)

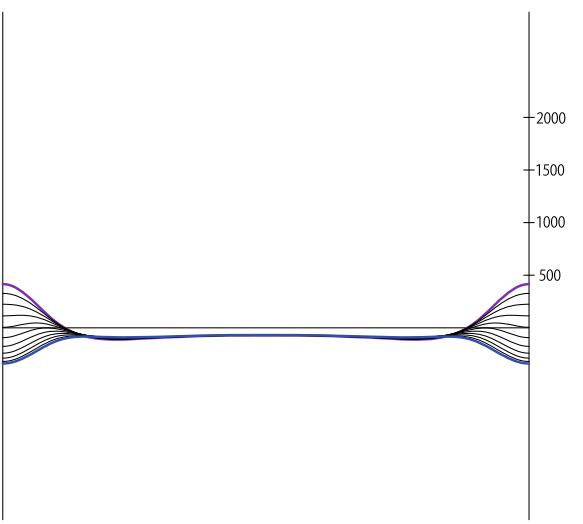

(d)

Fig. 16 From $P_{4,6}$ to $N_{4,6}$. The graph of the real part of $u$ at $P_{4,6}$ is drawn in red. The states in $N_{4,6}$ are drawn in blue. Graphs a-d are, respectively, $P \rightarrow Q, Q \rightarrow N, P \rightarrow M, M \rightarrow N$

Multiple precision arithmetic by Fujiwara [11] may be used. Application of multiple precision arithmetic to ill-posed problems was advocated by [12], and an application to a linear backward heat equation was reported in [17].

With these papers as models, we tried some numerical experiments to find that our problem is very troublesome. We therefore show our results on the following modest problem: Beginning from $P_{0,8}$, can we compute solutions backward towards $P_{0, k}$ for $k=7,6, \ldots$ ? Since we already know those solutions, we can check whether our method for computing nonlinear heat equation in a backward direction works or not by this experiment.

For this computation we tested two methods. One is that we use only exflib, [11], which allows us to use multiple precision arithmetic with as many digits as we like. The second method is to use exflib and the Fourier filter technique by Krasny [23] simultaneously. 


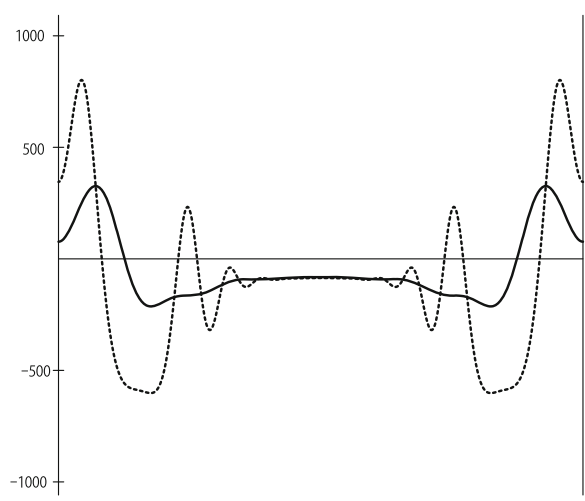

(a)

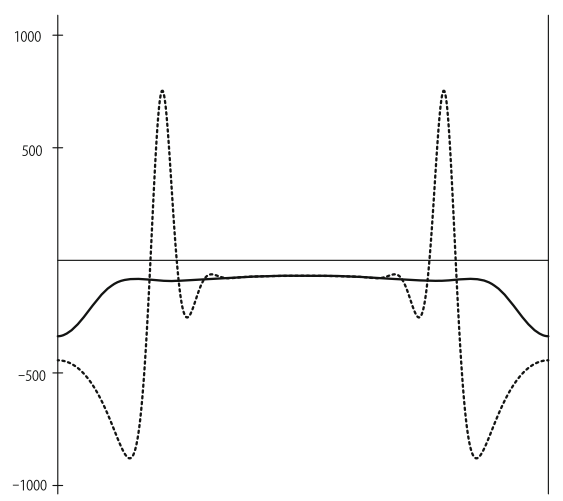

(b)

Fig. 17 a Two solutions at $P_{3.5,3}$. b Two solutions at $N_{4,6}=P_{5,7}$. The real parts of $u$ are drawn. The graph of broken line is the one from above, i.e., $P \rightarrow Q \rightarrow N$

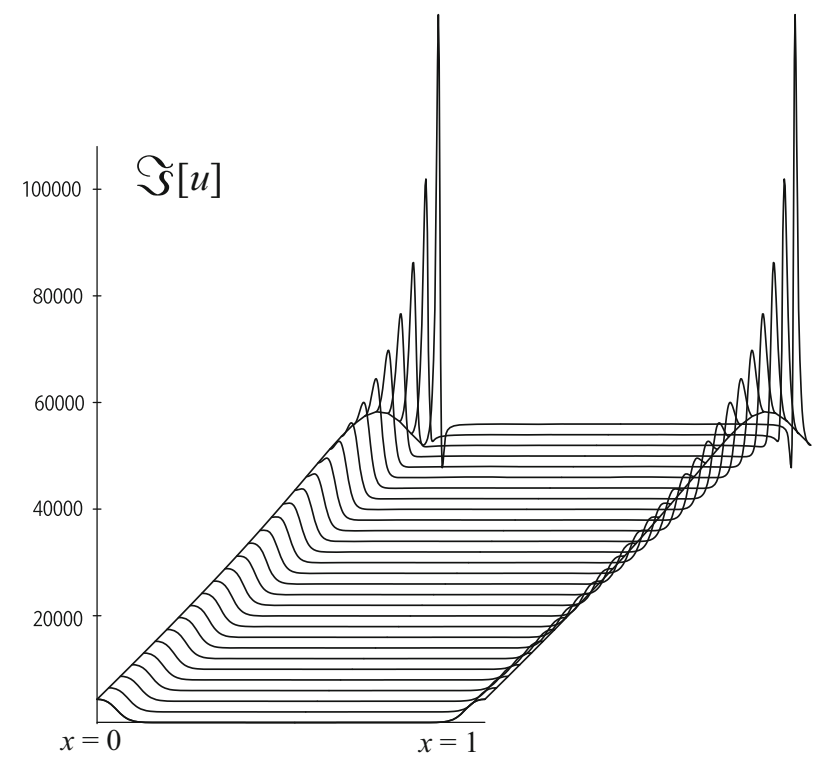

Fig. $18 \Im[u]$ on the line segment $\Re[t]=14.3 b,-4 b-\frac{7}{40} b \leq \Im[t] \leq-4 b$

We first computed the solution from $P_{0,8}$ to $P_{0,6}$ by using only exflib with 500 digits accuracy. (The computation with 1000 digits was carried out, but as far as the solution is well computed, no visible difference was noted, and we report our results with 500 digits.) The initial data is $u(0, x)=50(1-\cos 2 \pi x)$ and we use data at $P_{0, k}$ as computed by the method in the previous sections. The only difference is that, since we use the Fourier filters later, we take $K=512$. In Fig. 21a shows the real part of the solutions at $P_{0,6}, P_{0,7}$ and $P_{0,8}$ which are computed in the forward direction, and Fig. 21b shows the one at $P_{0,7}$, which is computed by exflib only (without Fourier filter) in the backward direction from $P_{0,8}$. Numerical instability is obviously occurring. 
Fig. 19 The region that two solutions agree (green) and disagree (red) (color figure online)

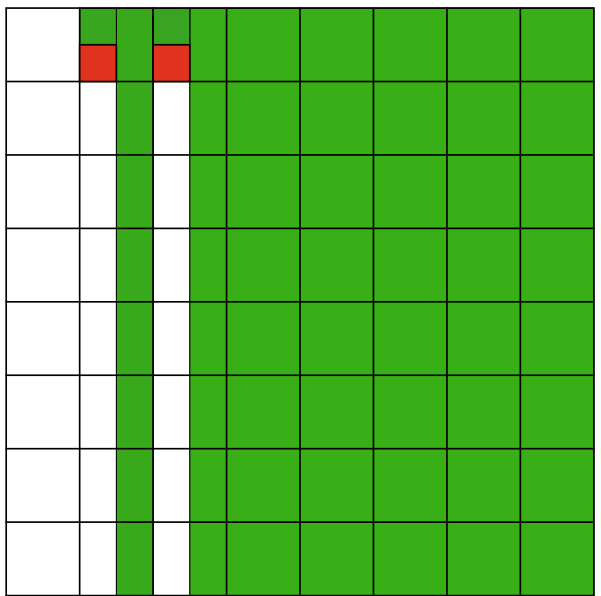

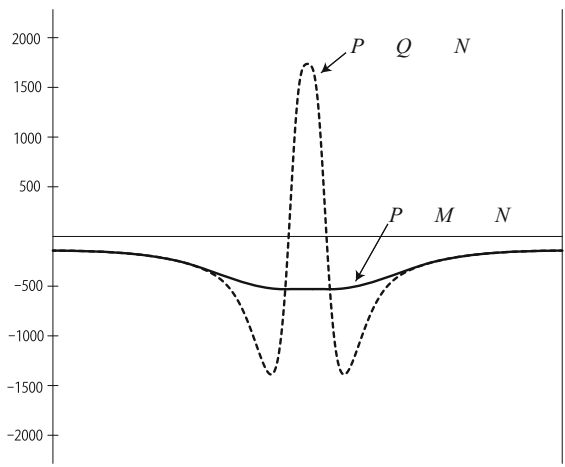

(a)

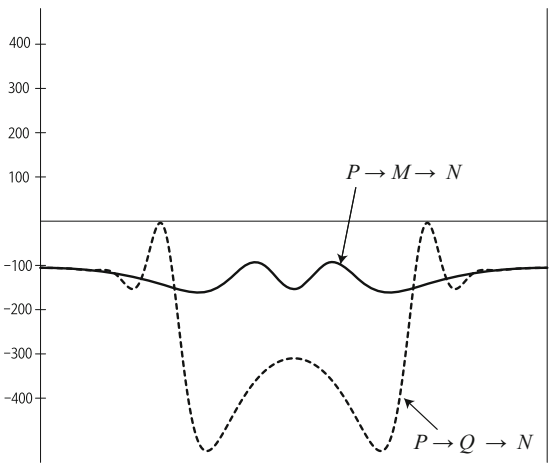

(b)

Fig. 20 The graphs of the real part of the two solutions at the right lower corners of the red squares in Fig. 19. a The red square on the left hand side. $\mathbf{b}$ The red square on the right hand side (color figure online)

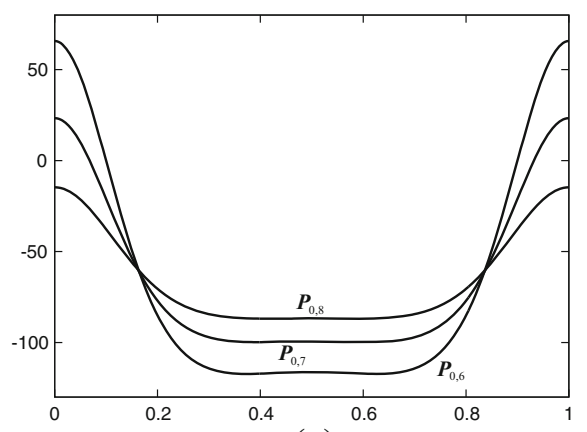

(a)

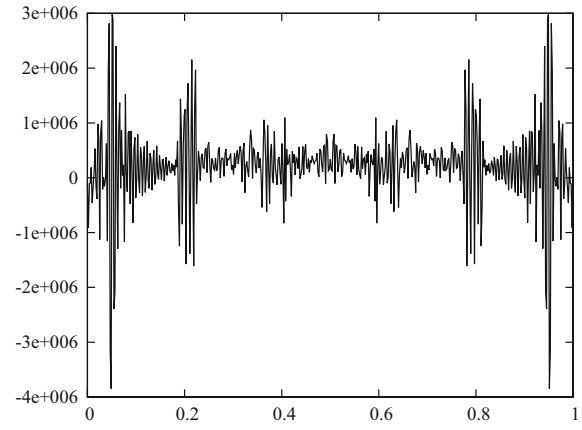

(b)

Fig. 21 a The real part of the solutions computed in the forward direction of $P_{0,6} \rightarrow P_{0,7} \rightarrow P_{0,8}$. b shows the one at $P_{0,7}$ computed in the backward direction from $P_{0,8}$ with no Fourier filter 


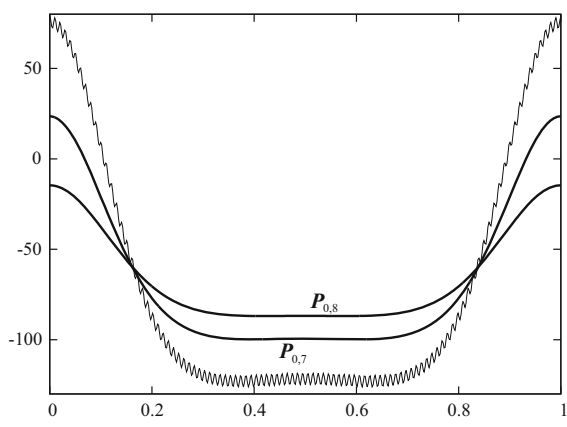

(a)

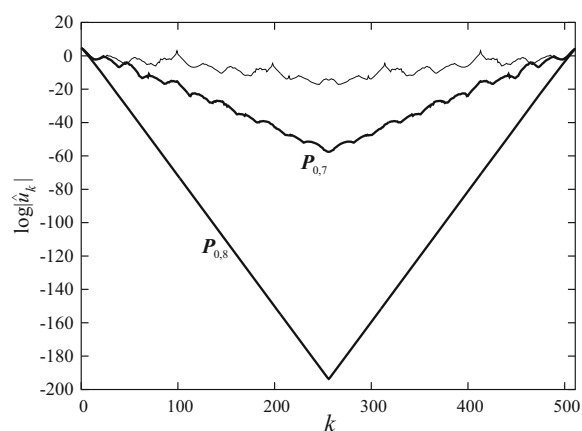

(b)

Fig. 22 Starting from $P_{0,8}$ with filter level $\varepsilon=10^{-12}$, a the real part of the solutions, at $P_{0,8}, P_{0,7}$, and slightly before reaching $P_{0,6}$. b The logarithm of $\left|\hat{u}_{k}\right|$

We now add the Fourier filter in the following way. We first choose a positive constant $\varepsilon$ and call it a filter level. At the end of each time step, a Fast Fourier Transform (FFT) is performed, namely from $\left\{u_{0}, u_{1}, \ldots, u_{K-1}\right\}$ we define $\left\{\hat{u}_{0}, \hat{u}_{1}, \ldots, \hat{u}_{K-1}\right\}$ by

$$
\hat{u}_{k}=K^{-1 / 2} \sum_{j=0}^{K-1} u_{j} \exp (-2 \pi \mathrm{i} j k / K) \quad(\mathrm{i}=\sqrt{-1}, k=0,1, \ldots, K-1) .
$$

Note that $\hat{u}_{k}$ with $k$ larger than $K / 2$ corresponds to the negative wavenumber $-(K-k)$. If the amplitude $\left|\hat{u}_{k}\right|$ of any mode $k$ is less than the filter level $\varepsilon$, then $\hat{u}_{k}$ is reset to zero. After having applied this rule on $\left\{\hat{u}_{k}\right\}$, we take the inverse FFT

$$
u_{k}=K^{-1 / 2} \sum_{j=0}^{K-1} \hat{u}_{j} \exp (2 \pi \mathrm{i} j k / K) \quad(k=0,1, \ldots K-1) .
$$

With this new $\left\{u_{k}\right\}$ we proceed to the next time step.

As is shown in Fig. 22, filtered with $\varepsilon=10^{-12}$, the solution at $P_{0,7}$ can be computed backward from $P_{0,8}$ and it matches well the one at $P_{0,7}$ in Fig. 21a. But thenceforth numerical instability becomes conspicuous before reaching $P_{0,6}$. Figure $22 \mathrm{~b}$ shows the logarithm of the absolute values of discrete Fourier coefficients as a function of the wavenumbers.

In order to continue further calculations, the filter level $\varepsilon$ must be taken larger. Figure 23a, b shows the results filtered at the level $10^{-5}$ and $10^{-4}$, respectively. $\varepsilon=10^{-5}$ is not, but $10^{-4}$ is enough for regularizing the solution to reach $P_{0,6}$. The solutions in (b) can be regarded to agree with those in Fig. 21a, since the relative errors of the $L^{2}$-norm (the left hand side of (9)) at $P_{0,7}$ and $P_{0,6}$ are less than $10^{-4}$ and $10^{-3}$ respectively.

These preliminary computations demonstrate that the accumulation of rounding error seems to be enormous in our problem, occurring in much larger scale than in the 


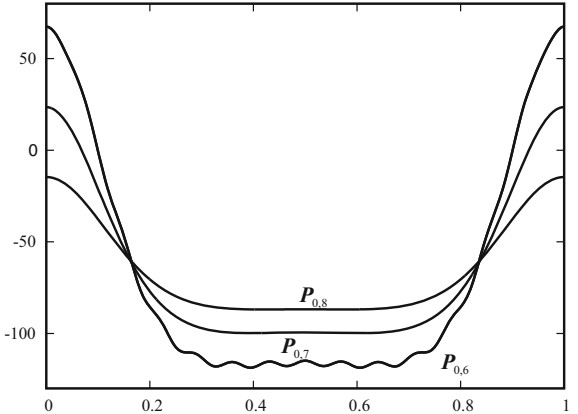

(a)

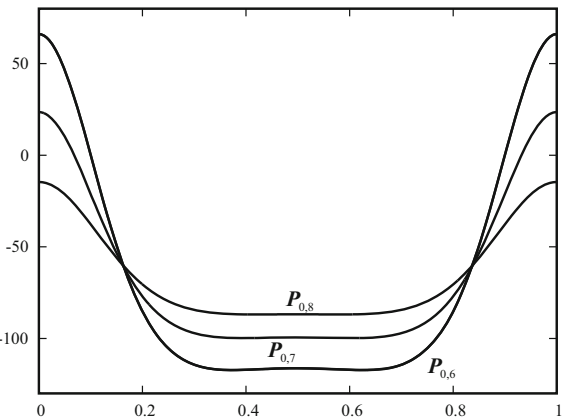

(b)

Fig. 23 a Filter level $\varepsilon=10^{-5}$. b Filter level $\varepsilon=10^{-4}$. These solutions are almost the same as those of Fig. 21a

linear backward heat equation. We therefore stop here and leave this problem to the future study.

\section{Concluding remarks}

It is likely that for many initial data which we tested in the present paper the solution has one and only one singularity on the real axis. The Riemann surface as the domain of definition of the solution is rather big. Below the "cut" $T<t<\infty$, branching points exist. On the other hand there seems to be no singularities on the imaginary axis. Although we cannot prove it, we believe that all the singularities are branch points and no isolated singularity is admitted anywhere in the Riemann surface.

Thus we may summarize our conclusion as the following two conjectures:

1. The analytic function defined by our nonlinear heat equation has branching singularities and only branching singularities, unless it is constant in $x$;

2. Our nonlinear Schrödinger equation (10) is globally well-posed for any real initial data, small or large.

The next task should be to study the relationship between the nature of the blowup on the real axis and branching singularities lying on the Riemann surface. This problem is left to the study in the future.

Acknowledgments We received important advice from many friends. We are indebted to Prof. Yoshio Tsutsumi, whose advice about the nonlinear Schrödinger equation helped improve our paper very much. Advice by Prof. Yuusuke Iso and Dr. Hiroshi Fujiwara for the use of multiple precision arithmetic is highly acknowledged. Prof. Krasny's advice improved our computation in Sect. 6. We express our deepest gratitude to those friends. Last but not least, we thank the referee for his/her critical reading and advice.

Open Access This article is distributed under the terms of the Creative Commons Attribution 4.0 International License (http://creativecommons.org/licenses/by/4.0/), which permits unrestricted use, distribution, and reproduction in any medium, provided you give appropriate credit to the original author(s) and the source, provide a link to the Creative Commons license, and indicate if changes were made. 


\section{References}

1. Caflisch, R.E.: Singularity formation for complex solutions of the 3D incompressible Euler equations. Phys. D 67, 1-18 (1993)

2. Cazenave, T.: Semilinear Schrödinger Equations. American Mathematical Society, Providence (2003)

3. Chan, T.F., Lee, D., Shen, L.: Stable explicit schemes for equations of the Schrödinger type. SIAM J. Numer. Anal. 23, 274-281 (1986)

4. Chen, Y.-G.: Asymptotic behaviours of blowing-up solutions for finite difference analogue of $u_{t}=$ $u_{x x}+u^{1+\alpha}$. J. Fac. Sci. Univ. Tokyo Sect. IA 33, 541-574 (1986)

5. Chen, Y.-G.: Blow-up solutions to a finite difference analogue of $u_{t}=u_{x x}+u^{1+\alpha}$ in $N$-dimensional ball. Hokkaido Math. J. 21, 447-474 (1992)

6. Cho, C.-H.: On the finite difference approximation for blow-up solutions of the porous medium equation with a source. Appl. Numer. Math. 65, 1-26 (2013)

7. Cho, C.-H., Hamada, S., Okamoto, H.: On the finite difference approximation for a parabolic blow-up problem. Japan J. Ind. Appl. Math. 24, 131-160 (2007)

8. Cho, C.-H., Okamoto, H.: Further remarks on asymptotic behavior of the numerical solutions of parabolic blow-up problems. Methods Appl. Anal. 14, 213-226 (2007)

9. Deng, K., Levine, H.A.: The role of critical exponents in blow-up theorems: the sequel. J. Math. Anal. Appl. 243, 85-126 (2000)

10. Fila, M., Matano, H.: Blow-up in Nonlinear Heat Equations from the Dynamical Systems Point of View, in Handbook of Dynamical Systems, vol. 2. North-Holland, Amsterdam (2002)

11. Fujiwara, H.: exflib. http://www-an.acs.i.kyoto-u.ac.jp/ fujiwara/exflib/index.html

12. Fujiwara, H., Iso, Y.: Numerical challenge to ill-posed problems by fast multiple-precision system. Theor. Appl. Mech. 50, 419-424 (2001)

13. Guo, J.-S., Ninomiya, H., Shimojo, M., Yanagida, E.: Convergence and blow-up of solutions for a complex-valued heat equation with a quadratic nonlinearity. Trans. Am. Math. Soc. 365, 2447-2467 (2013)

14. Hayashi, N., Naumkin, P.I.: On the quadratic nonlinear Schrödinger equation in three space dimensions. Int. Math. Res. Notices, 115-132 (2000)

15. Hayashi, N., Naumkin, P.I.: A quadratic nonlinear Schrödinger equation in one space dimension. J. Differ. Equ. 186, 165-185 (2002)

16. Hayashi, N., Naumkin, P.I.: Remark on the existence and large time asymptotics of solutions for the quadratic NLS. Nonlinear Anal. 74, 6950-6964 (2011)

17. Iijima, K.: Numerical solution of backward heat equation problems by a high order lattice-free finite difference method. J. Chin. Inst. Eng. 27, 611-620 (2004)

18. Kato, T.: Quasi-linear equations of evolution, with applications to partial differential equations. Springer Lect. Notes Math. 448, 25-70 (1975)

19. Kato, T.: Nonlinear Schrödinger equations. Schrödinger Operators. Springer Lect. Notes Phys. 345, 218-263 (1989)

20. Kimura, Y., Zawadzki, I., Aref, H.: Vortex motion, sound radiation, and complex time singularities. Phys. Fluids A 2, 214-219 (1990)

21. Kimura, Y.: Parametric motion of complex-time singularity toward real collapse. Phys. D 46, 439-448 (1990)

22. Kimura, Y., Pelz, R.B.: Search for complex singularities in Navier-Stokes turbulence. In: Kida, S. (ed.) Unstable and Turbulent Motion of Fluid, pp. 91-101. World Scientific, Singapore (1993)

23. Krasny, R.: Desingularization of periodic vortex sheet roll-up. J. Comput. Phys., 292-313 (1986)

24. Kuroda, S.T., Suzuki, T.: A time-dependent method for computing eigenvalues of Schrödinger operators. Japan J. Ind. Appl. Math. 7, 231-253 (1990)

25. Levine, H.A.: The role of critical exponents in blow-up theorems. SIAM Rev. 32, 262-288 (1990)

26. Masuda, K.: Blow-up of solutions of some nonlinear diffusion equations. In: Fujita, H., Lax, P.D., Strang, G. (eds.) Nonlinear Partial Differential Equations in Applied Sciences: Proceedings of the US-Japan Seminar, Tokyo, pp. 119-131. North-Holland, Amsterdam (1983)

27. Masuda, K.: Analytic solutions of some nonlinear diffusion equations. Math. Z. 187, 61-73 (1984)

28. Pauls, W., Matsumoto, T., Frische, U., Bae, J.: Nature of complex singularities for the $2 \mathrm{D}$ Euler equations. Phys. D 219, 40-59 (2006)

29. Quittner, P., Souplet, P.: Superlinear Parabolic problems, Blow-up, Global Existence and Steady States. Birkhäuser, Basel (2007) 
30. Sasaki, T.: A second-order time-discretization scheme for a system of nonlinear Schrödinger equations. Proc. Japan Acad. Ser. A 90, 15-20 (2014)

31. Siegel, M., Caflisch, R.E.: Calculation of complex singular solutions to the 3D incompressible Euler equations. Phys. D 238, 2368-2379 (2009)

32. Strikwerda, J.C.: Finite Difference Schemes and Partial Differential Equations. Wadsworth \& Brooks, Pacific Grove (1989)

33. Sulem, C., Sulem, P.-L., Frisch, H.: Tracing complex singularities with spectral method. J. Comput. Phys. 50, 138-161 (1983)

34. Tsutsumi, Y.: $L^{2}$-solutions for nonlinear Schrödinger equations and nonlinear groups. Funlcialaj Ekvacioj 30, 115-125 (1987)

35. Yanagida, E.: Blow-up of solutions of the nonlinear heat equations. In: Yanagida, E. (ed.) Blow-up and Aggregation, pp. 1-50. University of Tokyo Press, Tokyo (2006). (in Japanese)

36. Zhang, J.: On the finite time behaviour for nonlinear Schrödinger equations. Commun. Math. Phys. 162, 249-260 (1994) 